\title{
Interactive comment on "Evaluation of the interactive stratospheric ozone (03v2 module) for the E3SM version 2 Earth System Model" by Qi Tang et al.
}

\section{Anonymous Referee \#1}

Received and published: 19 October 2020

The Tang et al. manuscript presents a revised approach to parameterizing the distribution of ozone for use in the model radiation schemes. The approach is based on the widely used Linoz scheme in the stratosphere, but replaces the specified ozone previously used in the troposphere with the ozone tracer predicted by Linoz, subject to an imposed lower boundary condition of $30 \mathrm{ppb}$ near the surface. The advantage of the revised approach is a more consistent specification of ozone around the tropopause, particularly in the case where the tropopause is higher than the climatological average implicit in the specified tropospheric ozone climatology. The two approaches are compared by implementing them in the US Department of Energy's Energy Exascale Earth System Model (E3SMv1) for AMIP-type simulations using specified sea-surface

Printer-friendly version

Discussion paper 
temperatures for $1995-2014$.

In general, the manuscript presents the results in a scientifically valid way and I have only minor comments. I would be a bit critical of the presentation of the material, however. The manuscript spends some time comparing various aspects of the ozone climatology that results from the two parameterizations installed in E3SMv1 against observations, here I am referring to the results shown in Figures $1-4$. It is only a bit later that the impacts of the revised ozone parameterization on the model dynamics is discussed and the reader realizes that the differences discussed during the first part of the paper are not just the results of the revised ozone parameterization but also result from significant changes in the model dynamics. The authors could provide some overview of the situation earlier in the manuscript. I would also offer some criticism of the fact that there are significant differences in the ozone cross section shown in Figure 7, particularly in the lower stratosphere. While the problems with the original ozone parameterization in the vicinity of the tropopause are easy to imagine, there is no specific comparison against observations to show that the revised ozone parameterization produces a more correct ozone mixing ratio in this region.

\section{Minor Corrections:}

The title: I might suggest moving the word 'module' outside of the brackets so that it is 'stratospheric ozone (O3v2) module for...'

Page 1, Lines 21-23: The sentence 'As expected, SST forcing does not match the observed quasi-biennial oscillation...' could more clearly convey information. Having a free-running atmosphere forced only by observed SSTs does not match the observed QBO. It is not clear if there is a QBO spontaneously generated in E3SMv1 and the problem is that the model QBO is not in phase with the observed QBO, or whether there is not a QBO at all in E3SMv1. Page 6, Lines 4 - 8: The O3v1 control is described as being one of the three AMIP simulations forced with prescribed SSTs and sea-ice. The CMIP6 AMIP experiment that is part of the DECK is specified as running from

Printer-friendly version

Discussion paper
Interactive comment 
1979 - 2014 using observed SSTs and sea-ice, so the wording used here can be a bit confusing because AMIP refers to several runs of different length using specified SSTs and sea-ice. Perhaps 'AMIP-type' would be less confusing. The text also does not specifically mentioning whether the SSTs/sea-ice are from observations. The source of the SSTs/sea-ice should also be stated here. HadISSTs, I assume?

Page 6, Lines $14-17$ : Coming back to the use of the word AMIP to mean any run with specified SSTs/sea-ice, here the text refers to a pair of AMIP simulations where one of them uses SSTs increased by $4 \mathrm{~K}$. I understand what you mean by AMIP, but AMIP has a specific usage in CMIP and it is being used considerably more loosely here, particularly when SSTs are increased by $4 \mathrm{~K}$. I would suggest moving away from referring to all the runs as 'AMIP simulations'. Page 6, Line 17: Do you modify the sea-ice at all for the case where the SSTs are uniformly increased by $4 \mathrm{~K}$ ? This is not an objection to any particular treatment of sea-ice, just that it would be helpful to better understand how the simulation was set up.

Page 9, Line 12: For the STD/SCO shown in Figure 2, there are two time periods plotted up. Is the Taylor diagram in Figure 3 for the two period combined or only one of them?

Page 9, Lines 21 -23: On the improvement in the RMS error in the standard deviation of the SCO for O3v2, have you considered just internal variability? Both O3v1 and O3v2 are from free-running simulations of the E3SMv1 and they do show some regions of significant difference between the two period sampled.

Page 11, Lines 1 - 12: It is interesting that for both versions of E3SM the RMS for the annual average standard deviation (Figure 3c) is larger than for any of the individual months. It is a bit more difficult to see, but the annual average for $\mathrm{UCI}$ is within the cloud of points formed by the individual months. Do you have any ideas for why the RMS of the annual average standard deviation for E3SM would be larger than for any of the months?

Printer-friendly version

Discussion paper
Interactive

comment 
Page 11, Lines 23 - 27: Figure 5 shows the time evolution of zonal average ozone for O3v1 and the difference O3v2 - O3v1. There is the differences in Northern hemisphere ozone, with O3v1 having much larger columns and can be more easily seen in Figure 1 , but given the year-to-year variability in dynamics a straight year-to-year difference plot is significantly affected by the dynamical variability. I would suggest the authors replace the O3v2 - O3v1 difference with a plot of the O3v2 column as this would more clearly show the ozone hole behaviour in O3v2.

Page 14, Lines $1-4$ : The cross-section of ozone changes between O3v1 and O3v2 shown in Figure 7 show absolute differences which makes it difficult to assess the correctness of the statement 'with O3v2 having about $20 \%$ less ozone in the lower stratosphere, but hardly any change in the troposphere.... I would ask the authors to consider adding cross-section plots of the percentage differences - if not as a replacement for the current panels in Figure 7 then as an additional figure in the appendix?

Page 34, Caption to Figure A1. Here a list of five years with weak Antarctic ozone depletion is given for which the differences between O3v1 and O3v2 are calculated. If both simulations are free-running and have the ozone interactive with the model, shouldn't the years with weak ozone be different between the two simulations?

Interactive comment on Geosci. Model Dev. Discuss., https://doi.org/10.5194/gmd-2020-293, 2020. 